\title{
Existence and Multiplicity of Positive Solutions for Singular $p$-Laplacian Equations
}

\author{
Haishen Lü and Yi Xie
}

Abstract. Positive solutions are obtained for the boundary value problem

$$
\left\{\begin{array}{rlrl}
-\Delta_{p} u & =\lambda\left(u^{\beta}+\frac{1}{u^{\alpha}}\right) & & \text { in } \Omega \\
u>0 & & \text { in } \Omega \\
u & =0 & & \text { on } \partial \Omega
\end{array}\right.
$$

where $\Delta_{p} u=\operatorname{div}\left(|\nabla u|^{p-2} \nabla u\right), 1<p<N, N \geq 3, \Omega \subset \mathbb{R}^{N}$ is a bounded domain, $0<\alpha<1$ and $p-1<\beta<p^{*}-1\left(p^{*}=\frac{N p}{N-p}\right)$ are two constants, $\lambda>0$ is a real parameter. We obtain that Problem (*) has two positive weakly solutions if $\lambda$ is small enough.

Keywords. $p$-Laplacian, positive solution, critical point theory

Mathematics Subject Classification (2000). Primary 35J20, secondary 35J25

\section{Introduction}

In this paper we study the singular boundary value problem

$$
\left\{\begin{aligned}
-\Delta_{p} u & =\lambda\left(u^{\beta}+\frac{1}{u^{\alpha}}\right) & & \text { in } \Omega \\
u & >0 & & \text { in } \Omega \\
u & =0 & & \text { on } \partial \Omega,
\end{aligned}\right.
$$

where $\Delta_{p} u=\operatorname{div}\left(|\nabla u|^{p-2} \nabla u\right), 1<p<N, N \geq 3, \Omega \subset \mathbb{R}^{N}$ is a bounded domain, $0<\alpha<1$ and $p-1<\beta<p^{*}-1\left(p^{*}=\frac{N p}{N-p}\right)$ are two constants, $\lambda>0$ is a real parameter.

Haishen Lü: Department of Applied Mathematics, Hohai University, Nanjing 210098, China; haishen2001@yahoo.com.cn

Yi Xie: Department of Applied Mathematics, Hohai University, Nanjing 210098, China.

The research is supported by NNSF of China (10301033). 
Definition 1.1. A function $u \in W_{0}^{1, p}(\Omega)$ is called a positive weakly solution of Problem (1), if $u(x)>0$ for $x \in \Omega$ and

$$
\int_{\Omega}\left(|\nabla u|^{p-2} \nabla u, \nabla \varphi\right) d x=\lambda \int_{\Omega} u^{\beta} \varphi d x+\lambda \int_{\Omega} \frac{\varphi}{u^{\alpha}} d x \quad \text { for all } \varphi \in W_{0}^{1, p}(\Omega)
$$

holds.

In the pioneering work [1], A. Ambrosetti, H. Brezis and G. Cerami investigated the problem

$$
\left\{\begin{aligned}
-\Delta u & =\lambda u^{a}+u^{b} & & \text { in } \Omega \\
u & >0 & & \text { in } \Omega \\
u & =0 & & \text { on } \partial \Omega
\end{aligned}\right.
$$

with $0<a<1<b$. In the succeeding work [2], the above problem is extended to the $p$-Laplacian by A. Ambrosetti, J. G. Azorero and I. Peral. Motivated by this, this paper attempt to improve the above results to the singular $p$-Laplacian equation, i.e., $-1<a<0$. We must point out that since the functional of (1) fails to be Frechet differentiable in $\Omega$, critical point theory where $[1,2]$ have used could not be applied to obtain the existence of solutions. So the method in $[1,2]$ could not be used. So, it is very difficult to find existence and multiplicity of positive solutions for Problem (1).

The existence of solutions to the elliptic equation

$$
\left\{\begin{aligned}
-\Delta u & =\frac{f(x)}{u^{\gamma}} & & \text { in } \Omega \\
u & =0 & & \text { on } \partial \Omega
\end{aligned}\right.
$$

on a smooth domain $\Omega \subset \mathbb{R}^{N}$ has been extensively studied (cf. $[5,7,8,11,12]$ and their references). For bounded $\Omega$, in [7] it is shown that Problem (2) with $0<\gamma<1$ has a unique positive weakly solution in $H_{0}^{1}(\Omega)$ if $p(x)$ is a nonnegative nontrivial function in $L^{2}(\Omega)$. For the general problem

$$
\left\{\begin{aligned}
-\Delta u & =\frac{\sigma}{u^{\gamma}}+\lambda u^{\beta} & & \text { in } \Omega \\
u & =0 & & \text { on } \partial \Omega
\end{aligned}\right.
$$

It is worth mentioning that, in [10] the existence of a unique positive solution in the cases when $\beta=1$ and $0<\beta<1$ (the sub-linear problem) has been proved. On the other hand, in [4], Y. Sun, S. Wu and Y. Long have proved that Problem (3) has at least one positive weakly solution $u \in H_{0}^{1}(\Omega)$ for all $\lambda>0$ and $\sigma \in\left(0, \sigma^{*}\right]$.

Our goal in this paper is to prove that Problem (1) has two positive weakly solutions for all $\lambda$ small enough. In this paper, critical point theory could not be 
applied to obtain the existence of solutions since the associate functional fails to be Frechet differentiable in $\Omega$. We mainly rely on the Ekeland's variational principle [6] and careful estimates inspirsed by Lair-Shaker [7] and Tarantello [3].

We work on the Sobolev space $W_{0}^{1, p}(\Omega)$ equipped with the norm $\|u\|=$ $\left(\int_{\Omega}|\nabla u|^{p} d x\right)^{\frac{1}{p}}$. For $u \in W_{0}^{1, p}(\Omega)$ we define $I: W_{0}^{1, p}(\Omega) \rightarrow R$ by

$$
I(u)=\frac{1}{p} \int_{\Omega}|\nabla u|^{p} d x-\frac{\lambda}{\beta+1} \int_{\Omega}|u|^{\beta+1} d x-\frac{\lambda}{1-\alpha} \int_{\Omega}|u|^{1-\alpha} d x .
$$

On the other hand, $L^{p}(\Omega)$ denote Lebesgue's spaces, the norm in $L^{p}$ is denoted by $\|\cdot\|_{p} ; C_{1}, C_{2}, \cdots$ denote (possibly different) positive constants. Our main results is the following:

Theorem 1.2. Let $\Omega$ be a bounded domain in $\mathbb{R}^{N}, N \geq 3$. Let $0<\alpha<1$, $p<\beta+1<p^{*}$. Then there exists $\lambda_{0}>0$ such that for all $\lambda \in\left(0, \lambda_{0}\right)$ Problem (1) possesses at least two positive weakly solutions $u_{1}(\cdot), u_{2}(\cdot) \in W_{0}^{1, p}(\Omega)$ and

$\int_{\Omega}\left|\nabla u_{i}\right|^{p-2} \nabla u_{i} \cdot \nabla \varphi d x=\lambda \int_{\Omega} u_{i}^{\beta} \varphi d x+\lambda \int_{\Omega} \frac{\varphi}{u_{i}^{\alpha}} d x \quad$ for all $\varphi \in W_{0}^{1, p}(\Omega), i=1,2$.

Moreover, $u_{1}$ is a local minimizer of $I$ in $W_{0}^{1, p}(\Omega)$ with $I\left(u_{1}\right)<0$; and $u_{2}$ is a minimizer of $I$ on $\Lambda_{-}\left(\Lambda_{-}\right.$is defined behind) with $I\left(u_{2}\right) \geq 0$.

Remark 1.3. The conclusion of Theorem 1.2 can be extended to the case of the more general problem

$$
\left\{\begin{aligned}
-\Delta_{p} u & =\mu\left(\frac{f(x)}{u^{\rho}}+g(x) u^{\tau}\right) & & \text { in } \Omega \\
u & >0 & & \text { in } \Omega \\
u & =0 & & \text { on } \partial \Omega
\end{aligned}\right.
$$

where $f, g: \Omega \rightarrow R$ are two given non-negative and non-trival function in $L^{p}(\Omega)$.

Remark 1.4. When $N=1$, the type of equations has been studied by Agarwal and O'Regan [9] who proved that the equation

$$
\left\{\begin{aligned}
-\left(\left|u^{\prime}\right|^{q-2} u^{\prime}\right)^{\prime} & =\varsigma\left(\frac{1}{u^{\alpha_{1}}}+u^{\beta_{1}}+1\right) \text { for } 0<t<1,1<q<\infty \\
u(0) & =u(1)=0
\end{aligned}\right.
$$

where $\alpha_{1}>0, \beta_{1}>q-1$ and $0<\varsigma<\frac{2^{q}}{3}\left(\frac{q}{q-1+\alpha}\right)^{q-1}$, has two solutions $u_{1}$, $u_{2} \in C[0,1] \cap C^{1}(0,1)$ with $u_{1}>0, u_{2}>0$ on $(0,1)$ and $\left\|u_{1}\right\|_{\infty}<1<\left\|u_{2}\right\|_{\infty}$. 


\section{Preliminary lemmas}

Let us define

$$
\Lambda=\left\{u \in W_{0}^{1, p}(\Omega):\|u\|^{p}-\lambda\|u\|_{\beta+1}^{\beta+1}-\lambda \int_{\Omega}|u|^{1-\alpha}=0\right\} .
$$

It is easy to see that $\Lambda \backslash\{0\}$ is a Nehari manifold, see [14]. Notice that if $u$ is a weak of (1), then $u \in \Lambda$. For the sake of the convenience, we record

$$
\begin{array}{ll}
A=\frac{p-1+\alpha}{\beta+\alpha}, & B=\frac{\beta-p+1}{\beta+\alpha}, \quad D=\frac{p+\alpha-1}{\beta+1-p} \\
E=\frac{p^{*}-\beta-1}{p^{*}(\beta+1)}, & F=\frac{\beta+\alpha}{\beta+1} .
\end{array}
$$

Further, we define $G: W_{0}^{1, p}(\Omega) \rightarrow R$ by

$$
G(u)=A\|u\|^{p}-\lambda\|u\|_{\beta+1}^{\beta+1} .
$$

In succession, let

$$
\begin{aligned}
\Lambda_{+} & =\{u \in \Lambda: G(u)>0\} \\
\Lambda_{0} & =\{u \in \Lambda: G(u)=0\} \\
\Lambda_{-} & =\{u \in \Lambda: G(u)<0\} .
\end{aligned}
$$

For the sake of the convenience, we list some inequalities which we will use in the next section. By Sobolev's embedding Theorem, we have

$$
\begin{gathered}
\|u\|_{p} \leq C_{0}\|u\| \quad \forall u \in W_{0}^{1, p}(\Omega) \\
\|u\|_{p^{*}} \leq\left(\frac{1}{S}\right)^{\frac{1}{p}}\|u\| \quad \forall u \in W_{0}^{1, p}(\Omega),
\end{gathered}
$$

where $C_{0}>0$ is a constant and $S>0$ is the best Sobolev constant. By Hölder inequalities we have

$$
\begin{aligned}
\|u\|_{\beta+1} \leq|\Omega|^{E}\|u\|_{p^{*}} & \forall u \in W_{0}^{1, p}(\Omega) \\
\int_{\Omega}|u|^{1-\alpha} d x \leq|\Omega|^{F}\|u\|_{\beta+1}^{1-\alpha} & \forall u \in W_{0}^{1, p}(\Omega) .
\end{aligned}
$$

By (5) and (6), we have

$$
\|u\|_{\beta+1} \leq C_{1}\|u\| \quad \forall u \in W_{0}^{1, p}(\Omega)
$$

where $C_{1}=|\Omega|^{E}\left(\frac{1}{S}\right)^{\frac{1}{p}}$. By (7) and (8), we have

$$
\int_{\Omega}|u|^{1-\alpha} d x \leq C_{2}\|u\|^{1-\alpha} \quad \forall u \in W_{0}^{1, p}(\Omega)
$$

where $C_{2}=|\Omega|^{F+E(1-\alpha)} S^{\frac{\alpha-1}{p}}$. 
Lemma 2.1. Let

$$
\lambda_{1}=\left(\frac{A^{\delta} B S^{\frac{1}{p}}}{|\Omega|^{E+F}}\right)^{\frac{1}{D}},
$$

where $A, B, D, E, F, S$ are defined in (4) and (5). Then, for all $\lambda \in\left(0, \lambda_{1}\right)$, we have the following conclusions:

1. For every $u \in \Lambda, u \nRightarrow 0$, then $G(u) \neq 0$, (i.e., $\Lambda_{0}=\{0\}$ );

2. $\Lambda_{-}$is closed in $W_{0}^{1, p}(\Omega)$.

Proof. 1. Suppose, by contradiction that there exists some $u \in \Lambda, u \not \equiv 0$ such that $G(u)=0$. Then

$$
\|u\|_{\beta+1}^{\beta+1}=\frac{A}{\lambda}\|u\|^{p}
$$

So

$$
0=\|u\|^{p}-\lambda\|u\|_{\beta+1}^{\beta+1}-\lambda \int_{\Omega}|u|^{1-\alpha} d x=\|u\|^{p}-A\|u\|^{p}-\lambda \int_{\Omega}|u|^{1-\alpha} d x .
$$

Thus

$$
\int_{\Omega}|u|^{1-\alpha} d x=\frac{1-A}{\lambda}\|u\|^{p}=\frac{B}{\lambda}\|u\|^{p} .
$$

By (11) and (12) we have

$$
\frac{B}{\lambda}\|u\|^{p}\left(\frac{A}{\lambda}\right)^{D} \frac{\|u\|^{p D}}{\|u\|_{\beta+1}^{(\beta+1) D}}-\int_{\Omega}|u|^{1-\alpha} d x=0 .
$$

On the other hand, by (7) and (8) we have

$$
\begin{aligned}
\frac{B}{\lambda}\|u\|^{p}\left(\frac{A}{\lambda}\right)^{D} \frac{\|u\|^{p D}}{\|u\|_{\beta+1}^{(\beta+1) D}}-\int_{\Omega}|u|^{1-\alpha} d x & \geq \frac{B}{\lambda}\left(\frac{A}{\lambda}\right)^{D} \frac{S^{\frac{1}{p}}}{|\Omega|^{E}} \frac{\|u\|_{\beta+1}^{p D+p}}{\|u\|_{\beta+1}^{(\beta+1) D}}-|\Omega|^{F}\|u\|_{\beta+1}^{1-\alpha} \\
& =\left(\frac{A^{D} B}{\lambda^{1+D}} \frac{S^{\frac{1}{p}}}{|\Omega|^{E}}-|\Omega|^{F}\right)\|u\|_{\beta+1}^{1-\alpha} .
\end{aligned}
$$

If $0<\lambda<\lambda_{1}$, then $\frac{A^{D} B}{\lambda^{1+D}} \frac{S^{\frac{1}{p}}}{|\Omega|^{E}}-|\Omega|^{F}>0$. Thus

$$
\frac{B}{\lambda}\|u\|^{p} \cdot\left(\frac{A}{\lambda}\right)^{D} \frac{\|u\|^{p \sigma}}{\|u\|_{\beta+1}^{(\beta+1) D}}-\int_{\Omega}|u|^{1-\alpha} d x>0,
$$

which yields a contraction by (13). So $\Lambda_{0}=\{0\}$.

2. Let $\left\{u_{n}\right\} \subset \Lambda_{-}$be a sequence such that $u_{n} \rightarrow u_{0}$ in $W_{0}^{1, p}(\Omega)$. Then $u_{n} \rightarrow u_{0}$ in $L^{\beta+1}(\Omega)$ and $u_{0} \in \Lambda_{-} \cup \Lambda_{0}$. Now we prove $u_{0} \in \Lambda_{-}$. Suppose 
$u_{0} \in \Lambda_{0}$. Since $\Lambda_{0}=\{0\}$, it follows that $u_{0}=0$. On the other hand, for all $u \in \Lambda_{-}$,

$$
\frac{A}{\lambda} \leq \frac{\|u\|_{\beta+1}^{\beta+1}}{\|u\|^{p}}
$$

By (8), we have

$$
\frac{A S}{\lambda|\Omega|^{E p}} \leq\|u\|_{\beta+1}^{\beta+1-p}
$$

Thus

$$
\frac{A S}{\lambda|\Omega|^{E p}} \leq\left\|u_{n}\right\|_{\beta+1}^{\beta+1-p} \quad \text { for } n \in N
$$

Let $n \rightarrow \infty$, we have

$$
\frac{A S}{\lambda|\Omega|^{E p}} \leq\left\|u_{0}\right\|_{\beta+1}^{\beta+1-p}
$$

So $u_{0} \not \equiv 0$. Hence $u_{0} \in \Lambda_{-}$.

Lemma 2.2. Let

$$
\lambda_{2}=A^{\frac{D}{1-D}} \cdot B^{\frac{1}{1-D}} \cdot \frac{S}{|\Omega|^{p E+F}}
$$

where $A, B, D, E, F, S$ are defined in (4) and (5). If $0<\lambda<\lambda_{2}$, then for all $u \in W_{0}^{1, p}(\Omega), u \equiv 0$, there exists a unique $t^{+}=t^{+}(u)>0$ such that $t^{+} u \in \Lambda_{-}$.

Proof. For all $u \in W_{0}^{1, p}(\Omega), u \nRightarrow 0$, define $H:[0, \infty) \rightarrow(-\infty, \infty)$ by

$$
H(t)=t^{p-1+\alpha}\|u\|^{p}-\lambda t^{\beta+\alpha}\|u\|_{\beta+1}^{\beta+1} .
$$

Easy computations show that $H$ achieves its maximum at

$$
t_{0}=\left(\frac{A}{\lambda} \frac{\|u\|^{p}}{\|u\|_{\beta+1}^{\beta+1}}\right)^{\frac{1}{\beta+1-p}} .
$$

So

$$
H\left(t_{0}\right)=\left(\frac{A}{\lambda}\right)^{D} B \cdot\left[\frac{\|u\|^{p(\beta+\alpha)}}{\|u\|_{\beta+1}^{(\beta+1)(p+\alpha-1)}}\right]^{\frac{1}{\beta+1-p}} .
$$

If $\lambda \in\left(0, \lambda_{2}\right)$, then $\lambda|\Omega|^{F}\|u\|_{\beta+1}^{1-\alpha}<H\left(t_{0}\right)$. By (7), $\lambda \int|u|^{1-\alpha} d x \leq \lambda|\Omega|^{F}\|u\|_{\beta+1}^{1-\alpha}$. So $\lambda \int|u|^{1-\alpha} d x<H\left(t_{0}\right)$.

On the other hand, $H^{\prime}(t)<0$ for $t \in\left(t_{0}, \infty\right)$ and $\lim _{t \rightarrow+\infty} H(t)=-\infty$. So, there exists a unique $t^{+} \in\left(t_{0}, \infty\right)$ such that $H\left(t^{+}\right)=\lambda \int|u|^{1-\alpha} d x$, i.e., $\left\|t^{+} u\right\|^{p}-\lambda\left\|t^{+} u\right\|_{\beta+1}^{\beta+1}=\lambda \int_{\Omega}|t u|^{1-\alpha} d x$. So $t^{+} u \in \Lambda$. By

$$
H^{\prime}\left(t^{+}\right)=(p-1+\alpha)\left(t^{+}\right)^{p-2+\alpha}\|u\|^{p}-\lambda(\beta+\alpha)\left(t^{+}\right)^{\beta+\alpha-1}\|u\|_{\beta+1}^{\beta+1}<0,
$$

we have $G\left(t^{+} u\right)=\left(A\left\|t^{+} u\right\|^{p}-\lambda\left\|t^{+} u\right\|_{\beta+1}^{\beta+1}\right) \leq 0$. So $t^{+} u \in \Lambda_{-}$.

Remark 2.3. From Lemma 2.2 it follows that the set $\Lambda_{-}$is nonempty. 
Lemma 2.4. Given $u \in \Lambda_{-}$, then there exist $\varepsilon>0$ and a continuous function $f=f(w)>0, w \in W_{0}^{1, p}(\Omega),\|w\|<\varepsilon$, satisfying

$$
f(0)=1, \quad f(w)(u+w) \in \Lambda_{-} \text {for all } w \in W_{0}^{1, p}(\Omega),\|w\|<\varepsilon .
$$

Proof. Define $F: R \times W_{0}^{1, p}(\Omega) \rightarrow R$ as follows:

$$
F(t, w)=t^{p-1+\alpha}\|u+w\|^{p}-\lambda t^{\beta+\alpha}\|u+w\|_{\beta+1}^{\beta+1}-\lambda \int_{\Omega}|u+w|^{1-\alpha} d x .
$$

Since $u \in \Lambda_{-}(\subset \Lambda)$, it follows that $F(1,0)=0$ and

$$
F_{t}(1,0)=(p-1+\alpha)\|u\|^{p}-\lambda(\beta+\alpha)\|u\|_{\beta+1}^{\beta+1}<0,
$$

then we can apply the implicit function theorem at the point $(1,0)$ and obtain $\bar{\varepsilon}>0$ and a continuous function $f=f(w)>0, w \in W_{0}^{1, p}(\Omega),\|w\|<\bar{\varepsilon}$, satisfying $f(0)=1, F(f(w), w)=0$ for all $w \in W_{0}^{1, p}(\Omega),\|w\|<\bar{\varepsilon}$. Hence $f(w)(u+w) \in \Lambda$. Let $\varepsilon \in(0, \bar{\varepsilon})$ small enough, we have $f(w)(u+w) \in \Lambda_{-}$for all $w \in W_{0}^{1, p}(\Omega),\|w\|<\varepsilon$.

Lemma 2.5. Let

$$
\lambda_{3}=\left(\frac{\beta+1}{1-\alpha}\right)^{B} D^{B}|\Omega|^{F B} \frac{A S}{|\Omega|^{E p}} .
$$

Then, for all $\lambda \in\left(0, \lambda_{3}\right]$, the whole set $\Lambda_{-}$lies at the nonnegative level, that is $I(u) \geq 0$, for all $u \in \Lambda_{-}$.

Proof. We argue by contradiction. Suppose that exists $u_{0} \in \Lambda_{-} \subset \Lambda$ such that $I\left(u_{0}\right)<0$, i.e.,

$$
\frac{1}{p}\left\|u_{0}\right\|^{p}-\frac{\lambda}{\beta+1}\left\|u_{0}\right\|_{\beta+1}^{\beta+1}-\frac{\lambda}{1-\alpha} \int_{\Omega}\left|u_{0}\right|^{1-\alpha} d x<0 .
$$

By $u_{0} \in \Lambda$, we have $\left\|u_{0}\right\|^{p}=\lambda\left\|u_{0}\right\|_{\beta+1}^{\beta+1}+\lambda \int_{\Omega}\left|u_{0}\right|^{1-\alpha} d x$. By (17), we have

$$
\lambda\left(\frac{1}{p}-\frac{1}{\beta+1}\right)\left\|u_{0}\right\|_{\beta+1}^{\beta+1}+\lambda\left(\frac{1}{p}-\frac{1}{1-\alpha}\right) \int_{\Omega}\left|u_{0}\right|^{1-\alpha} d x<0,
$$

and by (7), we have

$$
\left\|u_{0}\right\|_{\beta+1}^{\beta+\alpha} \leq \frac{D(1+\beta)}{1-\alpha}|\Omega|^{F}
$$

By (14) (noticing $u_{0} \in \Lambda_{-}$), we have

$$
\left(\frac{A S}{\lambda|\Omega|^{E p}}\right)^{\frac{\beta+\alpha}{\beta+1-p}} \leq\left\|u_{0}\right\|_{\beta+1}^{\beta+\alpha} .
$$

If $0<\lambda<\lambda_{3}$, we have

$$
\left\|u_{0}\right\|_{\beta+1}^{\beta+\alpha} \leq \frac{D(1+\beta)}{1-\alpha}|\Omega|^{F}<\left(\frac{A S}{\lambda|\Omega|^{E p}}\right)^{\frac{\beta+\alpha}{\beta+1-p}} \leq\left\|u_{0}\right\|_{\beta+1}^{\beta+\alpha} .
$$

This is a contradiction. So $I\left(u_{0}\right) \geq 0$. 


\section{Proof of Theorem 1.2}

In this section, we prove that there exist $\lambda_{0}>0$ such that, for all $\lambda \in\left(0, \lambda_{0}\right)$, there exist at least two positive functions $u_{1}(\cdot), u_{2}(\cdot) \in W_{0}^{1, p}(\Omega)$ such that

$\int_{\Omega}\left|\nabla u_{i}\right|^{p-2} \nabla u_{i} \cdot \nabla \varphi d x=\lambda \int_{\Omega} u_{i}^{\beta} \varphi d x+\lambda \int_{\Omega} \frac{\varphi}{u_{i}^{\alpha}} d x \quad$ for all $\varphi \in W_{0}^{1, p}(\Omega), i=1,2$.

Moreover $u_{1}$ is a local minimizer of $I$ in $W_{0}^{1, p}(\Omega)$ with $I\left(u_{1}\right)<0$; and $u_{2}$ is a minimizer of $I$ on $\Lambda_{-}$.

Proof of Theorem 1.2. Using the inequalities (8) and (9), we have

$$
I(u) \geq \frac{1}{p}\|u\|^{p}-\lambda C_{3}\|u\|^{\beta+1}-\lambda C_{4}\|u\|^{1-\alpha}, \quad \forall u \in W_{0}^{1, p}(\Omega),
$$

where $C_{3}, C_{4}>0$ are positive constants. From this we readily find that there exists $\lambda_{4}>0$ such that for all $\lambda \in\left(0, \lambda_{4}\right]$ there are $r, a>0$ such that

(i) $I(u) \geq a$ for all $\|u\|=r$;

(ii) $I$ is bounded on $B_{r}=\left\{u \in W_{0}^{1, p}(\Omega):\|u\| \leq r\right\}$;

Let $\lambda_{0}=\min \left\{\lambda_{1}, \lambda_{2}, \lambda_{3}, \lambda_{4}\right\}$ where $\lambda_{i}(i=1,2,3)$ are the values found in (10), (15), (16), and $\lambda_{4}$ is defined as above. Next, we fix $\lambda \in\left(0, \lambda_{0}\right)$.

Existence of $u_{1}$. In view of of [6, Theorem 1.2] the infinimum of $I$ on $B_{r}$ can be achieved at a point $u_{1} \in B_{r}$. Note that, since $1-\alpha<1$, it follows that for every $v>0, I(t v)<0$ as $t>0$ small. So there exists $v_{1} \in B_{r}$ such that $I\left(v_{1}\right)<0$. Hence $I\left(u_{1}\right)=\inf _{u \in B_{r}} I(u) \leq I\left(v_{1}\right)<0$. This, together with (i), implies that $u_{1} \notin \partial B_{1}$. Hence $u_{1}$ is a local minimizer of $I$ in the $W_{0}^{1, p}$ topology. Clearly, $u_{1} \not \equiv 0$. Moreover, since $I(|u|)=I(u)$, we may assume that $u_{1} \geq 0$ in $\Omega$. Then, for any $\varphi \in W_{0}^{1, p}, \varphi \geq 0$,

$$
\begin{aligned}
0 \leq & I\left(u_{1}+t \varphi_{1}\right)-I\left(u_{1}\right) \\
= & \frac{1}{p}\left(\left\|u_{1}+t \varphi\right\|^{p}-\left\|u_{1}\right\|^{p}\right)+\frac{\lambda}{\beta+1}\left(\left\|u_{1}\right\|_{\beta+1}^{\beta+1}-\left\|u_{1}+t \varphi\right\|_{\beta+1}^{\beta+1}\right) \\
& +\frac{\lambda}{1-\alpha}\left(\int_{\Omega}\left|u_{1}\right|^{1-\alpha} d x-\int_{\Omega}\left|u_{1}+t \varphi\right|^{1-\alpha} d x\right) \\
\leq & \frac{1}{p}\left(\left\|u_{1}+t \varphi\right\|^{p}-\left\|u_{1}\right\|^{p}\right),
\end{aligned}
$$

i.e.,

$$
0 \leq \frac{1}{p} \int_{\Omega}\left(\left|\nabla\left(u_{1}+t \varphi\right)\right|^{p}-\left|\nabla u_{1}\right|^{p}\right) d x
$$

provided $t>0$ small enough. Dividing (18) by $t>0$ and passing to the limit as $t \rightarrow 0$, we derive

$$
\int_{\Omega}\left|\nabla u_{1}\right|^{p-2} \nabla u_{1} \cdot \nabla \varphi d x \geq 0 \quad \text { for } \varphi \in W_{0}^{1, p}(\Omega), \varphi \geq 0
$$


which means $u_{1} \in W_{0}^{1, p}(\Omega)$ satisfies in a weak sense that $-\Delta_{p} u_{1} \geq 0$ in $\Omega$. Since $u_{1} \geq 0, u_{1} \neq \equiv$, then the strong maximum principle yields

$$
u_{1}>0 \text { in } \Omega \text {. }
$$

On the other hand, from (18), we have

$$
\begin{aligned}
& \frac{\lambda}{1-\alpha}\left(\int_{\Omega}\left|u_{1}+t \varphi\right|^{1-\alpha} d x-\int_{\Omega}\left|u_{1}\right|^{1-\alpha} d x\right) \\
& \quad \leq \frac{1}{p}\left(\left\|u_{1}+t \varphi\right\|^{p}-\left\|u_{1}\right\|^{p}\right)-\frac{\lambda}{\beta+1}\left(\left\|u_{1}+t \varphi\right\|_{\beta+1}^{\beta+1}-\left\|u_{1}\right\|_{\beta+1}^{\beta+1}\right) .
\end{aligned}
$$

Dividing (19) by $t>0$ and passing to the limit, it follows that

$$
\begin{aligned}
& \frac{\lambda}{1-\alpha} \liminf _{t \rightarrow 0^{+}} \frac{\int_{\Omega}\left|u_{1}+t \varphi\right|^{1-\alpha} d x-\int_{\Omega}\left|u_{1}\right|^{1-\alpha} d x}{t} \\
& \leq \int_{\Omega}\left|\nabla u_{1}\right|^{p-2} \nabla u_{1} \cdot \nabla \varphi d x-\lambda \int_{\Omega} u_{1}^{\beta} \varphi d x .
\end{aligned}
$$

Observing

$$
\frac{1}{1-\alpha} \int_{\Omega} \frac{\left(u_{1}+t \varphi\right)^{1-\alpha}-u_{1}^{1-\alpha}}{t} d x=\int_{\Omega}\left(u_{1}+\theta t \varphi\right)^{-\alpha} \varphi d x
$$

where $\theta \rightarrow 0^{+}$as $t \rightarrow 0^{+}$and $\left(u_{1}+\theta t \varphi\right)^{-\alpha} \varphi \rightarrow u_{1}^{-\alpha} \varphi$ a.e. in $\Omega$ as $t \rightarrow 0^{+}$. Since $0 \leq\left(u_{1}+\theta t \varphi\right)^{-\alpha} \varphi$, for all $x \in \Omega$. By Fatou's Lemma, we have

$$
\frac{1}{1-\alpha} \liminf _{t \rightarrow 0^{+}} \int_{\Omega} \frac{\left(u_{1}+t \varphi\right)^{1-\alpha}-u_{1}^{1-\alpha}}{t} d x \geq \int_{\Omega} u_{1}^{-\alpha} \varphi d x .
$$

Combining (20) and (21), we have, for all $\varphi \in W_{0}^{1, p}(\Omega), \varphi \geq 0$,

$$
0 \leq \int_{\Omega}\left|\nabla u_{1}\right|^{p-2} \nabla u_{1} \cdot \nabla \varphi d x-\lambda \int_{\Omega} u_{1}^{\beta} \varphi d x-\lambda \int_{\Omega} u_{1}^{-\alpha} \varphi d x .
$$

On the other hand, there exists $\eta_{1} \in(0,1)$ such that $u_{1}+t u_{1} \in B_{r}$ for $|t| \leq \eta_{1}$. We define $h_{1}:\left[-\eta_{1}, \eta_{1}\right] \rightarrow R$ by $h_{1}(t) \equiv I\left((1+t) u_{1}\right)$. We have that $h_{1}(t)$ achieves its minimum at $t=0$. Therefore,

$$
\left.\frac{d h_{1}}{d t}\right|_{t=0}=\int_{\Omega}\left[\left|\nabla u_{1}\right|^{p}-\lambda u_{1}^{\beta+1}-\lambda u_{1}^{1-\alpha}\right] d x=0 .
$$

Therefore, $u_{1} \in \Lambda$.

We next prove that $u_{1}$ is a positive weakly solution. Suppose $\phi \in W_{0}^{1, p}(\Omega)$ and $\varepsilon>0$. Let $\Psi \equiv\left(u_{1}+\varepsilon \phi\right)^{+}$, where $\left(u_{1}+\varepsilon \phi\right)^{+}=\max \left\{u_{1}+\varepsilon \phi, 0\right\}$. Then 
$\Psi \in W_{0}^{1, p}(\Omega)$ and $\Psi \geq 0$. Inserting $\Psi$ into (22) and using (23) again, we infer that

$$
\begin{aligned}
0 \leq & \int_{\Omega}\left|\nabla u_{1}\right|^{p-2} \nabla u_{1} \cdot \nabla \Psi d x-\lambda \int_{\Omega} u_{1}^{\beta} \Psi d x-\lambda \int_{\Omega} u_{1}^{-\alpha} \Psi d x \\
= & \int_{\Omega \backslash \Omega_{\varepsilon}}\left[\left|\nabla u_{1}\right|^{p-2} \nabla u_{1} \cdot \nabla\left(u_{1}+\varepsilon \phi\right)-\lambda u_{1}^{\beta}\left(u_{1}+\varepsilon \phi\right)-\lambda u_{1}^{-\alpha}\left(u_{1}+\varepsilon \phi\right)\right] d x \\
= & \int_{\Omega}\left[\left|\nabla u_{1}\right|^{p}-\lambda u_{1}^{\beta+1}-\lambda u_{1}^{1-\alpha}\right] d x \\
& +\varepsilon \int_{\Omega}\left|\nabla u_{1}\right|^{p-2} \nabla u_{1} \cdot \nabla \phi d x-\lambda \varepsilon \int_{\Omega} u_{1}^{\beta} \phi d x-\lambda \varepsilon \int_{\Omega} u_{1}^{-\alpha} \phi d x \\
& -\int_{\Omega_{\varepsilon}}\left[\left|\nabla u_{1}\right|^{p-2} \nabla u_{1} \cdot \nabla\left(u_{1}+\varepsilon \phi\right)-\lambda u_{1}^{\beta}\left(u_{1}+\varepsilon \phi\right)-\lambda u_{1}^{-\alpha}\left(u_{1}+\varepsilon \phi\right)\right] d x \\
\leq & \varepsilon \int_{\Omega}\left[\left|\nabla u_{1}\right|^{p-2} \nabla u_{1} \cdot \nabla \phi-\lambda u_{1}^{\beta} \phi-\lambda u_{1}^{-\alpha} \phi\right] d x-\varepsilon \int_{\Omega_{\varepsilon}}\left|\nabla u_{1}\right|^{p-2} \nabla u_{1} \cdot \nabla \phi d x,
\end{aligned}
$$

where $\Omega_{\varepsilon}=\left\{x \in \Omega: u_{1}(x)+\varepsilon \phi(x)<0\right\}$. Since the measure of $\Omega_{\varepsilon}$ tends to zero as $\varepsilon \rightarrow 0$, it follows that $\int_{\Omega_{\varepsilon}}\left|\nabla u_{1}\right|^{p-2} \nabla u_{1} \cdot \nabla \phi d x \rightarrow 0$ as $\varepsilon \rightarrow 0$. Dividing by $\varepsilon$ and letting $\varepsilon \rightarrow 0$ therefore shows

$$
\int_{\Omega}\left[\left|\nabla u_{1}\right|^{p-2} \nabla u_{1} \cdot \nabla \phi-\lambda u_{1}^{\beta} \phi-\lambda u_{1}^{-\alpha} \phi\right] d x \geq 0 .
$$

Noting that $\phi$ is arbitrary, this holds equally for $-\phi$. So

$$
\int_{\Omega}\left[\left|\nabla u_{1}\right|^{p-2} \nabla u_{1} \cdot \nabla \phi-\lambda u_{1}^{\beta} \phi-\lambda u_{1}^{-\alpha} \phi\right] d x=0, \quad \text { for all } \phi \in W_{0}^{1, p}(\Omega) .
$$

Hence, $u_{1}$ is a positive weak solution of $(1)$ and $I\left(u_{1}\right)<0$

Next, we prove that (1) has another positive weakly solution $u_{2}$ such that $I\left(u_{2}\right)>0$. We first show that $I$ is coercive on $\Lambda$. Indeed, for $u \in \Lambda$, we have

$$
\|u\|^{p}-\lambda\|u\|_{\beta+1}^{\beta+1}-\lambda \int_{\Omega}|u|^{1-\alpha} d x=0
$$

By (24) and (9), we have

$$
\begin{aligned}
I(u) & =\frac{1}{p}\|u\|^{p}-\frac{\lambda}{\beta+1}\|u\|_{\beta+1}^{\beta+1}-\frac{\lambda}{1-\alpha} \int_{\Omega}|u|^{1-\alpha} d x \\
& \geq\left(\frac{1}{p}-\frac{1}{\beta+1}\right)\|u\|^{p}-\lambda C_{2}\left(\frac{1}{1-\alpha}-\frac{1}{\beta+1}\right)\|u\|^{1-\alpha} .
\end{aligned}
$$

So, $I$ is coercive on $\Lambda$. Since $\Lambda_{-}$is a closed set in $W_{0}^{1, p}(\Omega)$, we apply Ekeland's variational Principle to the minimization problem $\inf _{\Lambda_{-}} I$. It gives a minimizing sequence $\left\{w_{n}\right\} \subset \Lambda_{-}$with the following properties: 
(i) $I\left(w_{n}\right)<\inf _{\Lambda_{-}} I+\frac{1}{n}$;

(ii) $I(w) \geq I\left(w_{n}\right)-\frac{1}{n}\left\|w-w_{n}\right\|, \forall w \in \Lambda_{-}$.

Since $I(|u|)=I(u)$, we may assume that $w_{n} \geq 0$ in $\Omega$. By coerciveness, $\left\{w_{n}\right\}$ is bounded in $W_{0}^{1, p}(\Omega)$, i.e.,

$$
\left\|w_{n}\right\| \leq C_{5}, \quad n=1,2, \ldots,
$$

where $C_{5}>0$ is some constant independent on $n$. So there exists a subsequence (without loss of generality, suppose it is itself) and a function $u_{2} \geq 0$ such that

$$
\begin{array}{lll}
w_{n} \quad \rightarrow \quad u_{2} & \text { a.e. } x \in \Omega \\
w_{n} \stackrel{\text { strongly }}{\rightarrow} u_{2} & \text { in } L^{\beta+1} \\
w_{n} \stackrel{\text { weakly }}{\rightarrow} u_{2} & \text { in } W_{0}^{1, p} .
\end{array}
$$

On the other hand, by (14)

$$
\frac{A S}{\lambda|\Omega|^{E p}} \leq\left\|w_{n}\right\|_{\beta+1}^{\beta+1-p}
$$

so $u_{2} \not \equiv 0$. In addition, for the minimizing sequence $\left\{w_{n}\right\}$ there exists a suitable constant $C_{6}>0$ such that

$$
A\left\|w_{n}\right\|^{p}-\lambda\left\|w_{n}\right\|_{\beta+1}^{\beta+1} \leq-C_{6} \quad n=1,2, \ldots .
$$

Suppose, by contradiction, that for a subsequence, which is still denoted by $\left\{w_{n}\right\}$, we have

$$
A\left\|w_{n}\right\|^{p}-\lambda\left\|w_{n}\right\|_{\beta+1}^{\beta+1}=o(1) .
$$

Using $\left\{w_{n}\right\} \subset \Lambda_{-}$and (26), we have

$$
\begin{aligned}
I\left(w_{n}\right) & =\frac{1}{p}\left\|w_{n}\right\|^{p}-\frac{\lambda}{\beta+1}\left\|w_{n}\right\|_{\beta+1}^{\beta+1}-\frac{1}{1-\alpha}\left\|w_{n}\right\|^{p}+\frac{\lambda}{1-\alpha}\left\|w_{n}\right\|_{\beta+1}^{\beta+1} \\
& =-\frac{\beta+\alpha}{p(1-\alpha)} G\left(w_{n}\right)-\frac{\lambda(\beta+1-p)}{\beta+1}\left\|w_{n}\right\|_{\beta+1}^{\beta+1} \\
& \leq-\frac{\beta+\alpha}{p(1-\alpha)} G\left(w_{n}\right)-C_{7} \quad \text { for } n=1,2, \ldots,
\end{aligned}
$$

where $C_{7}>0$ is some constant independent of $n$. Passing to the limit as $n \rightarrow \infty$, we get $\lim _{n \rightarrow \infty} I\left(w_{n}\right) \leq-C_{7}$. This, together with $I\left(w_{n}\right) \geq \inf _{\Lambda_{-}} I(u)$ implies $\inf _{u \in \Lambda_{-}} I(u) \leq-C_{7}<0$, which is clearly impossible because from Lemma 2.5. It follows that $\inf _{u \in \Lambda_{-}} I(u) \geq 0$. 
For all $\varphi \in W_{0}^{1, p}(\Omega), \varphi \geq 0$, applying Lemma 2.4, with $u=w_{n}, w=t \varphi$, $t>0$ small, we find $f_{n}(t)=f_{n}(t \varphi)$ such that $f_{n}(0)=1$ and $f_{n}(t)\left(w_{n}+t \varphi\right) \in \Lambda_{-}$. Note that, since

$$
0=f_{n}^{p}(t)\left\|w_{n}+t \varphi\right\|^{p}-\lambda f_{n}^{\beta+1}\left\|w_{n}+t \varphi\right\|_{\beta+1}^{\beta+1}-f_{n}^{1-\alpha}(t) \int_{\Omega}\left(w_{n}+t \varphi\right)^{1-\alpha} d x
$$

and $0=\left\|w_{n}\right\|^{p}-\lambda\left\|w_{n}\right\|_{\beta+1}^{\beta+1}-\lambda \int_{\Omega} w_{n}^{1-\alpha} d x$, so

$$
\begin{aligned}
0= & f_{n}^{p}(t)\left\|w_{n}+t \varphi\right\|^{p}-\lambda f_{n}^{\beta+1}\left\|w_{n}+t \varphi\right\|_{\beta+1}^{\beta+1}-\lambda f_{n}^{1-\alpha}(t) \int_{\Omega}\left(w_{n}+t \varphi\right)^{1-\alpha} d x \\
& -\left\|w_{n}\right\|^{p}+\lambda\left\|w_{n}\right\|_{\beta+1}^{\beta+1}+\lambda \int_{\Omega} w_{n}^{1-\alpha} d x \\
= & \left(f_{n}^{p}(t)-1\right)\left\|w_{n}+t \varphi\right\|^{p}+\left(\left\|w_{n}+t \varphi\right\|^{p}-\left\|w_{n}\right\|^{p}\right) \\
& -\lambda\left(f_{n}^{\beta+1}-1\right)\left\|w_{n}+t \varphi\right\|_{\beta+1}^{\beta+1}-\lambda\left(\left\|w_{n}+t \varphi\right\|_{\beta+1}^{\beta+1}-\left\|w_{n}\right\|_{\beta+1}^{\beta+1}\right) \\
& -\lambda\left(f_{n}^{1-\alpha}-1\right) \int_{\Omega}\left(w_{n}+t \varphi\right)^{1-\alpha} d x-\lambda \int_{\Omega}\left[\left(w_{n}+t \varphi\right)^{1-\alpha}-w_{n}^{1-\alpha}\right] d x \\
\leq & \left(f_{n}^{p}(t)-1\right)\left\|w_{n}+t \varphi\right\|^{p}+\left(\left\|w_{n}+t \varphi\right\|^{p}-\left\|w_{n}\right\|^{p}\right) \\
& -\lambda\left(f_{n}^{\beta+1}-1\right)\left\|w_{n}+t \varphi\right\|_{\beta+1}^{\beta+1}-\lambda\left(\left\|w_{n}+t \varphi\right\|_{\beta+1}^{\beta+1}-\left\|w_{n}\right\|_{\beta+1}^{\beta+1}\right) \\
& -\lambda\left(f_{n}^{1-\alpha}-1\right) \int_{\Omega}\left(w_{n}+t \varphi\right)^{1-\alpha} d x .
\end{aligned}
$$

Dividing by $t>0$ and letting $t \rightarrow 0$, we infer that

$$
\begin{aligned}
0 \leq & p f_{n+}^{\prime}(0)\left\|w_{n}\right\|^{p}+p \int_{\Omega}\left|\nabla w_{n}\right|^{p-2} \nabla w_{n} \cdot \nabla \varphi d x \\
& -\lambda f_{n+}^{\prime}(0)(\beta+1)\left\|w_{n}\right\|_{\beta+1}^{\beta+1}-\lambda(\beta+1) \int_{\Omega} w_{n}^{\beta} \varphi d x-\lambda(1-\alpha) f_{n+}^{\prime}(0) \int_{\Omega} w_{n}^{1-\alpha} d x \\
= & f_{n+}^{\prime}(0)\left[p\left\|w_{n}\right\|^{p}-\lambda(\beta+1)\left\|w_{n}\right\|_{\beta+1}^{\beta+1}-\lambda(1-\alpha)\left\|w_{n}\right\|_{1-\alpha}^{1-\alpha}\right] \\
& +p \int_{\Omega}\left|\nabla w_{n}\right|^{p-2} \nabla w_{n} \cdot \nabla \varphi d x-\lambda(\beta+1) \int_{\Omega} w_{n}^{\beta} \varphi d x \\
= & f_{n+}^{\prime}(0)\left[(p+\alpha-1)\left\|w_{n}\right\|^{p}-\lambda(\beta+\alpha)\left\|w_{n}\right\|_{\beta+1}^{\beta+1}\right] \\
& +p \int_{\Omega}\left|\nabla w_{n}\right|^{p-2} \nabla w_{n} \cdot \nabla \varphi d x-\lambda(\beta+1) \int_{\Omega} w_{n}^{\beta} \varphi d x,
\end{aligned}
$$

i.e.,

$$
\begin{aligned}
0 \leq & f_{n+}^{\prime}(0)\left[(p+\alpha-1)\left\|w_{n}\right\|^{p}-\lambda(\beta+\alpha)\left\|w_{n}\right\|_{\beta+1}^{\beta+1}\right] \\
& +p \int_{\Omega}\left|\nabla w_{n}\right|^{p-2} \nabla w_{n} \cdot \nabla \varphi d x-\lambda(\beta+1) \int_{\Omega} w_{n}^{\beta} \varphi d x
\end{aligned}
$$


where $f_{n+}^{\prime}(0)=\lim _{t \rightarrow 0^{+}} \frac{f_{n}(t)-f_{n}(0)}{t}$. For the sake of simplicity, we assume henceforth that the right derivate of $f_{n}$ at $t=0$ exists. Indeed, if it doesn't exist, we let $t_{k} \rightarrow 0$ (instead of $\left.t \rightarrow 0\right), t_{k}>0$ is chosen in such a way that $f_{n}$ satisfies $q_{n}:=\lim _{k \rightarrow \infty} \frac{f_{n}\left(t_{k}\right)-f_{n}(0)}{t_{k}}$, then replace $f_{n+}^{\prime}(0)$ by $q_{n}$. We next prove that $f_{n+}^{\prime}(0) \neq \pm \infty$.

By (8) and (25)

$$
\begin{aligned}
&\left.\left|p \int_{\Omega}\right| \nabla w_{n}\right|^{p-2} \nabla w_{n} \cdot \nabla \varphi d x-\lambda(\beta+1) \int_{\Omega} w_{n}^{\beta} \varphi d x \mid \\
& \leq p\left\|w_{n}\right\|^{p-1}\|\varphi\|^{p}+\lambda(\beta+1)\left\|w_{n}\right\|_{\beta+1}^{\beta}\|\varphi\|_{\beta+1} \leq C_{8},
\end{aligned}
$$

where $C_{8}>0$ is a positive constant. For (27), (28) and (29), we know immediately that $f_{n+}^{\prime}(0) \neq+\infty$. Now we prove that $f_{n+}^{\prime}(0) \neq-\infty$. By contradiction, we assume that $f_{n+}^{\prime}(0)=-\infty$, and so for $t>0$ small there holds $f_{n}(t)<1$. Then

$$
\begin{aligned}
\left\|f_{n}(t)\left(w_{n}+t \varphi\right)-w_{n}\right\| & =\left(\int_{\Omega}\left|f_{n}(t)\left(\nabla w_{n}+t \nabla \varphi\right)-\nabla w_{n}\right|^{p} d x\right)^{\frac{1}{p}} \\
& =\left(\int_{\Omega}\left|\left(f_{n}(t)-1\right) \nabla w_{n}+t f_{n}(t) \nabla \varphi\right|^{p} d x\right)^{\frac{1}{p}} \\
& \leq\left[1-f_{n}(t)\right]\left\|w_{n}\right\|+t f_{n}(t)\|\varphi\|
\end{aligned}
$$

provided $t>0$ small. Thus, from (ii) we have $\frac{1}{n}\left\|w-w_{n}\right\| \geq I\left(w_{n}\right)-I(w)$. So

$$
\begin{aligned}
{\left[1-f_{n}(t)\right] \frac{\left\|w_{n}\right\|}{n}+t f_{n}(t) \frac{\|\varphi\|}{n} } \\
\quad \geq \frac{1}{n}\left\|f_{n}(t)\left(w_{n}+t \varphi\right)-w_{n}\right\| \\
\geq I\left(w_{n}\right)-I\left(f_{n}(t)\left(w_{n}+t \varphi\right)\right) \\
\quad=\frac{1}{p}\left\|w_{n}\right\|^{p}-\frac{\lambda}{\beta+1}\left\|w_{n}\right\|_{\beta+1}^{\beta+1}-\frac{\lambda}{1-\alpha} \int_{\Omega}\left|w_{n}\right|^{1-\alpha} d x-\frac{1}{p}\left\|f_{n}(t)\left(w_{n}+t \varphi\right)\right\|^{p} \\
\quad+\frac{\lambda}{\beta+1}\left\|f_{n}(t)\left(w_{n}+t \varphi\right)\right\|_{\beta+1}^{\beta+1}+\frac{\lambda}{1-\alpha} \int_{\Omega}\left|f_{n}(t)\left(w_{n}+t \varphi\right)\right|^{1-\alpha} d x
\end{aligned}
$$

Using

$$
-\frac{\lambda}{1-\alpha} \int_{\Omega}\left|w_{n}\right|^{1-\alpha} d x=-\frac{1}{1-\alpha}\left\|w_{n}\right\|^{p}+\frac{\lambda}{1-\alpha}\left\|w_{n}\right\|_{\beta+1}^{\beta+1}
$$

and

$$
\begin{aligned}
\frac{\lambda}{1-\alpha} \int_{\Omega}\left|f_{n}(t)\left(w_{n}+t \varphi\right)\right|^{1-\alpha} d x= & \frac{1}{1-\alpha} f_{n}^{p}(t)\left\|w_{n}+t \varphi\right\|^{p} \\
& -\frac{\lambda}{1-\alpha} f_{n}^{\beta+1}(t)\left\|w_{n}+t \varphi\right\|_{\beta+1}^{\beta+1}
\end{aligned}
$$


we have

$$
\begin{aligned}
& {\left[1-f_{n}(t)\right] \frac{\left\|w_{n}\right\|}{n}+t f_{n}(t) \frac{\|\varphi\|}{n} } \\
& \geq\left(\frac{1}{p}-\frac{1}{1-\alpha}\right)\left\|w_{n}\right\|^{p}-\left(\frac{\lambda}{\beta+1}-\frac{\lambda}{1-\alpha}\right)\left\|w_{n}\right\|_{\beta+1}^{\beta+1} \\
&-\left(\frac{1}{p}-\frac{1}{1-\alpha}\right) f_{n}^{p}(t)\left\|w_{n}+t \varphi\right\|^{p} \\
&+\lambda\left(\frac{1}{\beta+1}-\frac{1}{1-\alpha}\right) f_{n}^{\beta+1}(t)\left\|w_{n}+t \varphi\right\|_{\beta+1}^{\beta+1} \\
&= \frac{p+\alpha-1}{p(1-\alpha)}\left(\left\|w_{n}+t \varphi\right\|^{p}-\left\|w_{n}\right\|^{p}\right)+\frac{p+\alpha-1}{p(1-\alpha)}\left(f_{n}^{p}(t)-1\right)\left\|w_{n}+t \varphi\right\|^{p} \\
&-\lambda \frac{\beta+\alpha}{(\beta+1)(1-\alpha)} f_{n}^{\beta+1}(t)\left(\left\|w_{n}+t \varphi\right\|_{\beta+1}^{\beta+1}-\left\|w_{n}\right\|_{\beta+1}^{\beta+1}\right) \\
&-\lambda \frac{\beta+\alpha}{(\beta+1)(1-\alpha)}\left[f_{n}^{\beta+1}(t)-1\right]\left\|w_{n}\right\|_{\beta+1}^{\beta+1} .
\end{aligned}
$$

Dividing by $t>0$ and passing to the limit as $t \rightarrow 0$, we have

$$
\begin{aligned}
- & f_{n+}^{\prime}(0) \frac{\left\|w_{n}\right\|}{n}+\frac{\|\varphi\|}{n} \\
\geq & \frac{p+\alpha-1}{p(1-\alpha)} \int_{\Omega}\left|\nabla w_{n}\right|^{p-2} \nabla w_{n} \cdot \nabla \varphi d x+\frac{p+\alpha-1}{1-\alpha} f_{n+}^{\prime}(0)\left\|w_{n}\right\|^{p} \\
& -\frac{\beta+\alpha}{1-\alpha} \int_{\Omega}\left|w_{n}\right|^{\beta} \varphi d x-\lambda \frac{\beta+\alpha}{1-\alpha} f_{n+}^{\prime}(0)\left\|w_{n}\right\|_{\beta+1}^{\beta+1} \\
= & \frac{1}{1-\alpha}\left[(p+\alpha-1)\left\|w_{n}\right\|^{p}-\lambda(\beta+\alpha)\left\|w_{n}\right\|_{\beta+1}^{\beta+1}\right] f_{n+}^{\prime}(0) \\
& +\frac{1}{1-\alpha}\left[(p+\alpha-1) \int_{\Omega}\left|\nabla w_{n}\right|^{p-2} \nabla w_{n} \cdot \nabla \varphi d x-\lambda(\beta+\alpha) \int_{\Omega}\left|w_{n}\right|^{\beta} \varphi d x\right],
\end{aligned}
$$

i.e.,

$$
\begin{aligned}
\frac{\|\varphi\|}{n} \geq & \frac{1}{1-\alpha}\left[(p+\alpha-1)\left\|w_{n}\right\|^{p}-\lambda(\beta+\alpha)\left\|w_{n}\right\|_{\beta+1}^{\beta+1}+\frac{1-\alpha}{n}\left\|w_{n}\right\|\right] f_{n+}^{\prime}(0) \\
& +\frac{1}{1-\alpha}\left[(p+\alpha-1) \int_{\Omega}\left|\nabla w_{n}\right|^{p-2} \nabla w_{n} \cdot \nabla \varphi d x\right. \\
& \left.-\lambda(\beta+\alpha) \int_{\Omega}\left|w_{n}\right|^{\beta} \varphi d x\right] .
\end{aligned}
$$

By (25) and (27), there exist $N_{0}>0$ and $C_{9}>0$ (independent of $n$ ) such that, for $n \geq N_{0}$,

$$
\frac{1}{1-\alpha}\left[(p+\alpha-1)\left\|w_{n}\right\|^{p}-\lambda(\beta+\alpha)\left\|w_{n}\right\|_{\beta+1}^{\beta+1}+\frac{1-\alpha}{n}\left\|w_{n}\right\|\right] \leq-C_{9} .
$$


On the other hand, by (8) and (25), we have, for $n \geq N_{0}$,

$\left|\frac{1}{1-\alpha}\left[(p+\alpha-1) \int_{\Omega}\left|\nabla w_{n}\right|^{p-2} \nabla w_{n} \cdot \nabla \varphi d x-\lambda(\beta+\alpha) \int_{\Omega}\left|w_{n}\right|^{\beta} \varphi d x\right]\right| \leq C_{10}$, where $C_{10}>0$ (independent of $n$ ) is a suitable constant. By (30), it is impossible that $f_{n+}^{\prime}(0)=-\infty$. Furthermore, (28) and (30) imply that $\left|f_{n+}^{\prime}(0)\right| \leq$ $C_{11}$ for $n=1,2, \ldots$, where $C_{11}>0$ is a suitable constant.

Now we prove that $u_{2} \in \Lambda_{-}$is a positive weakly solution of (1). From condition (ii) we infer $\frac{1}{n}\left\|w-w_{n}\right\| \geq I\left(w_{n}\right)-I(w)$, i.e.,

$$
\begin{aligned}
& \frac{1}{n}\left[\left|f_{n}(t)-1\right|\left\|w_{n}\right\|+t f_{n}(t)\|\varphi\|\right] \\
& \geq \frac{1}{n}\left\|f_{n}(t)\left(w_{n}+t \varphi\right)-w_{n}\right\| \\
& \geq I\left(w_{n}\right)-I\left(f_{n}(t)\left(w_{n}+t \varphi\right)\right) \\
&= \frac{1}{p}\left\|w_{n}\right\|^{p}-\frac{\lambda}{\beta+1}\left\|w_{n}\right\|_{\beta+1}^{\beta+1}-\frac{\lambda}{1-\alpha} \int_{\Omega}\left|w_{n}\right|^{1-\alpha} d x \\
&-\frac{1}{p}\left\|f_{n}(t)\left(w_{n}+t \varphi\right)\right\|^{p}+\frac{\lambda}{\beta+1}\left\|f_{n}(t)\left(w_{n}+t \varphi\right)\right\|_{\beta+1}^{\beta+1} \\
&+\frac{\lambda}{1-\alpha} \int_{\Omega}\left|f_{n}(t)\left(w_{n}+t \varphi\right)\right|^{1-\alpha} d x \\
&=-\frac{f_{n}^{p}(t)-1}{p}\left\|w_{n}\right\|^{p}+\lambda \frac{f_{n}^{\beta+1}(t)-1}{\beta+1}\left\|w_{n}\right\|_{\beta+1}^{\beta+1}+\lambda \frac{f_{n}^{1-\alpha}(t)-1}{1-\alpha} \int_{\Omega}\left|w_{n}\right|^{1-\alpha} d x \\
&-\frac{f_{n}^{p}(t)}{p}\left(\left\|w_{n}+t \varphi\right\|^{p}-\left\|w_{n}\right\|^{p}\right)+\frac{\lambda}{\beta+1} f_{n}^{\beta+1}(t)\left(\left\|w_{n}+t \varphi\right\|_{\beta+1}^{\beta+1}-\left\|w_{n}\right\|_{\beta+1}^{\beta+1}\right) \\
&+\frac{\lambda}{1-\alpha} f_{n}^{1-\alpha}(t) \int_{\Omega}\left[\left(w_{n}+t \varphi\right)^{1-\alpha}-w_{n}^{1-\alpha}\right] d x .
\end{aligned}
$$

Dividing by $t>0$ and passing to the limit as $t \rightarrow 0$, this yields

$$
\begin{aligned}
\frac{1}{n}\left[\left|f_{n+}^{\prime}(0)\right|\left\|w_{n}\right\|+\right. & \|\varphi\|] \\
\geq & -f_{n+}^{\prime}(0)\left\|w_{n}\right\|^{p}+\lambda f_{n+}^{\prime}(0)\left\|w_{n}\right\|_{\beta+1}^{\beta+1}+\lambda f_{n+}^{\prime}(0) \int_{\Omega}\left|w_{n}\right|^{1-\alpha} d x \\
& -\int_{\Omega}\left|\nabla w_{n}\right|^{p-2} \nabla w_{n} \cdot \nabla \varphi d x+\lambda \int_{\Omega} w_{n}^{\beta} \varphi d x \\
& +\liminf _{t \rightarrow 0^{+}} \frac{\lambda}{1-\alpha} \int_{\Omega} \frac{\left(w_{n}+t \varphi\right)^{1-\alpha}-w_{n}^{1-\alpha}}{t} d x \\
= & -\int_{\Omega}\left|\nabla w_{n}\right|^{p-2} \nabla w_{n} \cdot \nabla \varphi d x+\lambda \int_{\Omega} w_{n}^{\beta} \varphi d x \\
& +\liminf _{t \rightarrow 0^{+}} \frac{\lambda}{1-\alpha} \int_{\Omega} \frac{\left(w_{n}+t \varphi\right)^{1-\alpha}-w_{n}^{1-\alpha}}{t} d x .
\end{aligned}
$$


Since $\left(w_{n}(x)+t \varphi(x)\right)^{1-\alpha}-w_{n}^{1-\alpha}(x) \geq 0$, for all $x \in \Omega, t>0$, then by Fatou's Lemma, we have

$$
\lambda \int_{\Omega} w_{n}^{1-\alpha} \varphi d x \leq \liminf _{t \rightarrow 0^{+}} \frac{\lambda}{1-\alpha} \int_{\Omega} \frac{\left(w_{n}+t \varphi\right)^{1-\alpha}-w_{n}^{1-\alpha}}{t} d x .
$$

So

$$
\begin{aligned}
\lambda \int_{\Omega} w_{n}^{-\alpha} \varphi d x \leq & \frac{1}{n}\left[\left|f_{n+}^{\prime}(0)\right|\left\|w_{n}\right\|+\|\varphi\|\right] \\
& +\int_{\Omega}\left|\nabla w_{n}\right|^{p-2} \nabla w_{n} \cdot \nabla \varphi d x-\lambda \int_{\Omega} w_{n}^{\beta} \varphi d x \\
\leq & \frac{C_{11} C_{5}+\|\varphi\|}{n}+\int_{\Omega}\left|\nabla w_{n}\right|^{p-2} \nabla w_{n} \cdot \nabla \varphi d x-\lambda \int_{\Omega} w_{n}^{\beta} \varphi d x .
\end{aligned}
$$

Let $n \rightarrow \infty$, we have

$$
\liminf _{n \rightarrow \infty} \lambda \int_{\Omega} w_{n}^{-\alpha} \varphi d x \leq \int_{\Omega}\left|\nabla u_{2}\right|^{p-2} \nabla u_{2} \cdot \nabla \varphi d x-\lambda \int_{\Omega} u_{2}^{\beta} \varphi d x
$$

then using once more Fatou's Lemma, we infer that, for all $\varphi \in W_{0}^{1, p}(\Omega), \varphi \geq 0$,

$$
\int_{\Omega}\left|\nabla u_{2}\right|^{p-2} \nabla u_{2} \cdot \nabla \varphi d x-\lambda \int_{\Omega} u_{2}^{\beta} \varphi d x-\lambda \int_{\Omega} w_{2}^{-\alpha} \varphi d x \geq 0,
$$

which means that $u_{2}$ satisfies $-\Delta_{p} u_{2} \geq 0$ in $\Omega$. Since $u_{2} \geq 0$ and $u_{2} \not \equiv 0$ in $\Omega$, then the strong maximum principle yields $u_{2}>0$ in $\Omega$. In particular, using (31) with $\varphi=u_{2}$, we infer that

$$
\left\|u_{2}\right\|^{p}-\lambda\left\|u_{2}\right\|_{\beta+1}^{\beta+1}-\lambda \int_{\Omega} u_{2}^{1-\alpha} d x \geq 0
$$

On the other hand, by weakly lower semi-continuity of the norm

$$
\left\|u_{2}\right\|^{p} \leq \lambda\left\|u_{2}\right\|_{\beta+1}^{\beta+1}+\lambda \int_{\Omega} u_{2}^{1-\alpha} d x
$$

So

$$
\left\|u_{2}\right\|^{p}=\lim _{n \rightarrow \infty}\left\|w_{n}\right\|^{p}=\lambda\left\|u_{2}\right\|_{\beta+1}^{\beta+1}+\lambda \int_{\Omega} u_{2}^{1-\alpha} d x
$$

Consequently

$$
w_{n} \stackrel{\text { strongly }}{\rightarrow} u_{2} \quad \text { in } W_{0}^{1, p}(\Omega)
$$

and $I\left(u_{2}\right)=\inf _{\Lambda_{-}} I$. Also from Lemma 2.1 , it follows that necessarily $u_{2} \in \Lambda_{-}$. Then, following the same arguments as in proving the existence of $u_{1}$ and using (31)-(32), we obtain $u_{2} \in \Lambda_{-}$is a positive weakly solution of (1). This completes the proof of Theorem 1.2. 


\section{References}

[1] Ambrosetti, A., Brezis, H. and Cerami, G., Combined effects of concave and convex nonlinearities in some elliptic problems. J. Funct. Anal. 122 (1994), $519-543$.

[2] Ambrosetti, A., Azorero, J. G. and Peral, I., Multiplicity results for some nonlinear elliptic equations. J. Funct. Anal. 137 (1996), 219 - 242.

[3] Tarantello, G., On nonhomogeneous elliptic equations involving critical Soblev exponent. Ann. Inst. H. Poincaré Anal. Non. Lineaire 9 (1992), 281 - 304.

[4] Sun, Y., Wu, S. and Long, Y., Combined effects of singular and superlinear nonlinearities in some singular boundary value problems. J. Diff. Equations 176 (2001), $511-531$.

[5] Crandall, M. G., Rabinowitz, P. H. and Tartar, L., On a Dirichlet problem with a singular nonlinearity. Comm. Partial Diff. Equations 2 (1977), $193-222$.

[6] Struwe, M., Variational Methods. Berlin: Springer 1990.

[7] Lair, A. V. and Shaker, A. W., Classical and weak solutions of a singular semilinear elliptic problem. J. Math. Anal. Appl. 211 (1997), 193 - 222.

[8] Lazer, A. C. and Mckenna, P. J., On a singular nonlinear elliptic boundary value problem. Proc. Amer. Math. Soc. 111 (1991), 721 - 730.

[9] Agarwal, P. P. and O'Regan, D., Singular Differential and Integral Equations with Applications. Kluwer 2003.

[10] Shi, J. and Yao, M., On a singular nonlinear semilinear elliptic problem. Proc. Roy. Soc. Edinburgh Sect. A 128 (1998), 1389 - 1401.

[11] Coclite, M. M., and Palmieri, G., On a singular nonlinear Dirichlet problems. Comm. Partial Diff. Equations 14 (1989), 1315 - 1327.

[12] Shaker, A. W., On singular semilinear elliptic equations. J. Math. Anal. Appl. 173 (1993), $222-228$.

[13] Diaz, J. I., Morel, J. M. and Oswald, L., An elliptic equation with singular nonlinearity. Comm. Partial Diff. Equations 12 (1987), 1333 - 1344.

[14] Willem, M., Minimax Theorems. Birkhäuser 1996.

Received November 29, 2005; revised December 21, 2005 